\title{
Perspective fonCtionnelle ET TYPES DE TEXTES \\ LES VARIANTES DE LA CONSTRUCTION TEXTUELLE DANS \\ EXERCICES DE STYLE DE RAYMOND QUENEAU ${ }^{1}$
}

\author{
Franciska SKUTTA \\ Université de Debrecen
}

\begin{abstract}
En): Based on the Prague School theory of functional sentence perspective, this essay examines the possible extension of FSP to the study of the information structure of the text. Following Firbas' theory concerning the existence of a basic distribution of communicative dynamism in language, and of deviations from it in actual utterances, the analysis of Raymond Queneau's Exercises in Style (1947) intends to show that each variant of the first, unmarked text (Notations) deviates from that model in a specific way with respect to theme - rheme opposition and thematic progression. These aspects of the particular texts appear to be determined by certain typological features, such as genre, style, or the speaker's attitude.
\end{abstract}

Keywords (En): functional sentence perspective; theme-rheme; thematic progression; text types; Queneau; Exercises in Style

Mots-clés (Fr): perspective fonctionnelle de la phrase ; thème-rhème ; progression thématique ; types de textes; Queneau ; Exercices de style

\section{Introduction}

Dans mon article, je me propose d'examiner les rapports entre un phénomène phrastique et un phénomène textuel, notamment entre la perspective fonctionnelle de la phrase (PFP; functional sentence perspective, FSP) et le «genre » d'un texte. L'hypothèse selon laquelle la perspective fonctionnelle de la phrase peut être déterminée en partie à un niveau supérieur, par les propriétés génériques d'un texte, sera vérifiée par l'étude des Exercices de style (Gallimard, 1947) de Raymond Queneau. En effet, cette œuvre originale, avec ses 99 variantes de la présentation d'un même contenu sémantique - une scène banale dans un autobus parisien -, constitue un terrain propice pour montrer qu'au-delà des variations lexico-grammaticales très nettes caractérisant le ton et les différents registres linguistiques, il doit y avoir une corrélation, peut-être moins évidente, entre le type d'un texte et sa configuration informationnelle.

Les travaux du Cercle Linguistique de Prague nous offrent la possibilité d'étendre le concept de la perspective fonctionnelle à l'examen du texte et de ses différentes réalisations. Je citerai en particulier un important article de Jan Firbas, publié en 1974, où l'auteur résume la question de la perspective fonctionnelle et de la dynamique communicative (DC; communicative dynamism, CD) de la façon suivante :

[...] the basic distribution of $\mathrm{CD}$ is a factor actually respected by language. In actual utterances, or utterance types, it tends to be either fully observed or to have the deviations duly signalled. [...] this is implemented by an interplay of means of FSP. The ways this interplay is

\footnotetext{
${ }^{1}$ Travail réalisé avec le soutien financier du Fonds National Hongrois pour les Recherches Scientifiques, OTKA, K 81913.
} 
implemented will naturally vary from language to language and lead to different hierarchies of word order principles. The ultimate point of departure, however, will remain the basic distribution of CD. (FIRBAS, $1974: 36)^{2}$

Certes, les observations de Firbas concernent le niveau de la phrase - ou de l'énoncé (utterance) effectivement produit par un locuteur -, mais certains termes clés de cette définition peuvent être appliqués également à l'étude de l'organisation informationnelle du texte, voire des types de textes. En effet, Firbas affirme à propos de la phrase énoncée qu'il existe une distribution fondamentale de la dynamique communicative, et que même si, dans les énoncés de divers types, il peut y avoir des déviations - dûment signalées -, le point de départ pour ces dernières reste toujours la distribution fondamentale des informations. Par analogie, on pourrait considérer dans certains cas - notamment chez Queneau - les types de textes comme des «déviations » par rapport à un texte modèle servant de point de départ pour décrire les variations dans l'alternance des informations connues et nouvelles (identifiables, entre autres, par l'ordre des termes), partant, dans la réalisation de la progression thématique caractérisant tel ou tel type de texte. Conformément à cette hypothèse, je vais examiner d'abord le texte de départ des Exercices de style, pour passer ensuite à l'analyse des phénomènes phrastiques et textuels dans les variantes.

\section{Le texte de départ}

C'est effectivement de cette manière que l'on peut concevoir l'étude des Exercices de style - ouvrage qui se prête à merveille à une telle analyse -, où le point de départ est constitué par le premier texte, servant de texte de référence, par rapport auquel les autres textes présentent autant de variations sur un même thème ${ }^{3}$ : l'aventure comique du jeune homme au cou trop long et au chapeau bizarre s'irritant contre un voyageur dans un autobus bondé, et plus tard, devant la gare Saint-Lazare, discutant avec un ami sur une insignifiante «question vestimentaire ». Pour pouvoir mieux montrer les déviations dans les variantes, je préfère citer ce texte dans son intégralité :

\section{Notations}

Dans l'S, à une heure d'affluence. Un type dans les vingt-six ans, chapeau mou avec cordon remplaçant le ruban, cou trop long comme si on lui avait tiré dessus. Les gens descendent. Le type en question s'irrite contre un voisin. Il lui reproche de le bousculer chaque fois qu'il passe quelqu'un. Ton pleurnichard qui se veut méchant. Comme il voit une place libre, se précipite dessus.

Deux heures plus tard, je le rencontre cour de Rome, devant la gare Saint-Lazare. Il est avec un camarade qui lui dit : «Tu devrais faire mettre un bouton supplémentaire à ton pardessus. » Il lui montre où (à l'échancrure) et pourquoi. (p. 5) ${ }^{4}$

Le titre Notations - et ce sera pareil pour les autres titres - préfigure le ton du texte : ici, un ton plus ou moins objectif, caractérisant un petit récit assez rapide,

\footnotetext{
${ }^{2}$ Cf. une formulation semblable dans FIRBAS (1971: 143).

3 J'emploie ici le mot «thème» dans son sens musical, comme dans l'expression «thème et variations ». Par la suite, le mot thème sera employé dans son sens linguistique, opposé à rhème.

${ }^{4}$ Les numéros de pages renvoient à l'édition de la Pléiade (2006). Pour toutes références, v. la Bibliographie.
} 
mené au présent à la manière d'un reportage, et dont le déroulement ne montre aucun trait «frappant » sur le plan de l'arrangement des informations. La scène est introduite par le cadre spatio-temporel (des compléments circonstanciels cadratifs, à fonction scénique), puis par la description du protagoniste, les deux segments textuels étant constitués par des propositions elliptiques, nominales. En revanche, les événements successifs sont racontés dans des phrases «canoniques », ou proches de la structure $S N$ sujet $+V(+S N$ complément $)$. Sur le plan des informations, cette structure syntaxique représente la division des phrases de ce texte essentiellement en deux parties : d'abord le thème, réalisé comme sujet grammatical et exprimant à la fois l'information connue et le point de départ vers d'autres informations ; ensuite le rhème - le verbe et/ou son complément - étant cet apport d'informations nouvelles et par là, le point d'aboutissement de l'énoncé. Cependant, dans quelques cas, on peut hésiter quant à la nature informationnelle d'un segment de phrase. Ainsi, les points de départ des deux phrases descriptives, Un type dans les vingt-six ans [...] et Ton pleurnichard [...] sont - pour des raisons différentes - rhématiques : la première des deux phrases commence en fait par un syntagme nominal indéfini, dont le référent, n'étant pas rattaché au contexte précédent, apparaît comme nouveau (bien que son évocation ne produise pas de véritable surprise ${ }^{5}$ étant donné le scénario d'un voyage en autobus), tandis que la deuxième phrase montre une structure informationnelle légèrement plus compliquée. Intégrée comme proposition principale dans une phrase complexe contenant une subordonnée relative, cette proposition peut être interprétée de deux façons: ou bien comme entièrement rhématique, puisque, prise en bloc, elle exprime des informations nouvelles à propos du thème élidé (par ex. : [le type parle d'un] ton pleurnichard), ou bien comme une succession de thème et de rhème dans une construction attributive où la copule a été supprimée ([le] ton [est] pleurnichard). Dans cette deuxième interprétation, la division est justifiée par le fait que le sujet défini évoque du connu grâce à l'anaphore associative qu'il établit avec son antécédent 'le type' (qui prononce des reproches) / 'les paroles du type'. Malgré ces cas peu conformes aux positions classiques du thème et du rhème (rhème en début de phrase, ou bien rhème suivant le thème sans insertion d'un verbe), la présentation du contenu sémantique reste sans emphase. Par ailleurs, la deuxième partie du récit a une structure informationnelle qui, dans l'ensemble, suit celle de la première, et qu'il n'est donc pas nécessaire de détailler ici.

Quant à la progression thématique, telle qu'elle se manifeste dans Notations, elle convient en gros à la structure du récit comme type de texte. La progression dominante, et assez fluide ici, est celle communément appelée progression à thème constant, où ce thème est représenté par le héros dont la permanence, à

\footnotetext{
${ }^{5}$ Certes, la « surprise » n'est pas un terme technique de l'étude de la PFP, mais on peut la considérer comme la partie la plus fortement rhématique de la dynamique communicative de la phrase. Sur le plan des informations, le «donné » et le «nouveau » sont, on le sait, des propriétés gradables. Dans la DC des phrases, et partant des textes, c'est l'élément rhématique non rattaché au contexte (le rhème propre) qui porte le poids informationnel le plus important, et qui peut éventuellement produire une «surprise » lors de la réception du texte, lorsque cet élément nouveau ne semble pas immédiatement compatible avec un cadre ou un scénario. Cf. pour «propriétés gradables » DANEŠ (1974 : 109), COMBETTES (1986 : 71).
} 
travers les événements successifs - autant de rhèmes - constitue le fil conducteur de l'histoire. En effet, une fois le protagoniste introduit sous forme rhématique (un type), il devient le thème - ou apparaît dans la sphère thématique - de la plupart des phrases: le syntagme nominal indéfini sujet est repris d'abord par un syntagme nominal défini sujet, en anaphore fidèle (le type en question), ensuite par une chaîne pronominale coréférentielle, en fonction de sujet et de complément d'objet, direct ou indirect ( $i l, l e, i l,[i l], l e, i l, l u i, l u i)$. À cela s'ajoute l'évocation de ce même référent dans le discours direct par le pronom personnel tu, qui le fait figurer dans le rôle du destinataire des paroles citées par le narrateur. Des types de progression secondaires pour ce texte apparaissent pour éviter la monotonie, telles la progression à thèmes dérivés (d'un hyperthème), dans les descriptions d'objets statiques (le chapeau, le cou et, en fin de compte, le type), ou la progression linéaire, le rhème devenant immédiatement thème (un camarade qui) ${ }^{6}$; on relève quelques ruptures thématiques (un type, les gens; le je du narrateur, latent jusquelà), qui sont malgré tout compatibles avec la scène décrite ; enfin, le changement de niveau d'énonciation (récit du narrateur - paroles d'un personnage), dûment signalé par la forme du discours direct, ne gêne pas non plus le déroulement du texte et l'interprétation des informations véhiculées.

\section{Les variantes}

Dans l'ensemble des Exercices de style, les 98 variantes - autant de « déviations » par rapport au texte de départ - forment souvent des groupes, ou pour ainsi dire, des faisceaux, où les textes successifs se caractérisent et s'opposent entre eux selon un même critère linguistique, stylistique ou générique. Ainsi trouve-t-on, par exemple, des groupements de textes où ce critère est constitué par les temps verbaux; dans d'autres cas, ce sont des structures syntaxiques variées, des registres linguistiques, des langues étrangères mêlées au français, des genres, littéraires ou non, plus ou moins codés, ou des figures de rhétorique (surtout phonétiques) sophistiquées. Cependant, l'ordre des textes n'obéit pas à un principe rigoureux ; sauf pour les ensembles fermés - tels les cinq textes ayant pour thématique centrale les cinq sens -, il arrive plusieurs fois qu'une succession de textes apparentés soit interrompue pour laisser la place momentanément à des textes d'un autre groupe, ce qui permet d'éviter une trop grande rigidité à cette œuvre essentiellement ludique.

\section{Jeux phonétiques}

Dans les variantes, les modifications - qui se manifestent à différents niveaux de la construction textuelle - n'ont pas toutes la même pertinence pour produire des changements notables dans l'organisation des informations; aussi ces variantes-là ne seront-elles pas examinées. En effet, plus d'un quart des textes des Exercices de style sont ainsi des variantes obtenues par divers jeux phonétiques, comme, à titre d'exemple, Anagrammes; Aphérèses - Syncopes - Apocopes, et leurs inverses : Prosthèses - Épenthèses - Paragoges ; Lipogramme, Métathèses,

\footnotetext{
${ }^{6}$ Pour ces trois types principaux de la progression thématique (thematic progression), ainsi que les combinaisons de ces types, cf. DANEŠ (1974: 114-123).
} 
Javanais, etc. Or ces procédés, déformant les mots dans leurs aspects phonétique et graphique (par addition, suppression ou permutation de sons et de lettres), les privent en même temps de leur signification, et par conséquent, de leur valeur communicative, de sorte que dans ces textes, le problème de la perspective fonctionnelle de la phrase ne se pose même pas. De plus, la restitution des mots dans leur intégrité n'ajoute rien à l'étude de la perspective fonctionnelle, car de toute façon, ces jeux phonétiques ne changent pas l'ordre des termes ni, bien sûr, l'arrangement des informations par rapport au texte de départ. Néanmoins, certaines déformations phonétiques - tout en créant des «mots » inexistants dans le vocabulaire du français - permettent une analyse sémantique et informationnelle. Il en est ainsi de Composition de mots, où l'on rencontre notamment des unités obtenues par la réunion hors norme de deux à trois mots, et qui peuvent entretenir des rapports divers avec les expressions originelles dans Notations. Il arrive certes que la perspective fonctionnelle de base soit maintenue malgré les «monstres » lexicaux, comme pour cette phrase de départ : Le type en question s'irrite contre un voisin (p. 5) devenant Lequel dit à un quelconquanonyme [...] (p. 13), mais dans d'autres cas, le nouveau terme hybride change radicalement le statut syntaxique et informationnel des termes originaux. Ainsi, le syntagme prépositionnel devant la gare Saint-Lazare - rattaché seulement en apposition supprimable au complément de lieu cour de Rome dans Notations - est transformé ici en un «verbe » ayant un poids phonétique et informationnel assez important pour fonctionner comme le début du rhème d'une subordonnée relative attributive: je le revis qui placesaint-lazarait avec un $X[\ldots]$ (p. 13).

\section{Types de textes}

Pour en venir aux nombreuses variantes du texte de départ qui ne sont pas dominées par des propriétés phonétiques récurrentes, on trouve qu'elles constituent un ensemble assez hétérogène de textes appartenant à des types divers, ces types étant toujours annoncés d'ailleurs par les titres. Certes, la notion de type de texte sera employée ici dans un sens assez flou ${ }^{7}$, comprenant soit des genres codés, illustrés par Sonnet, Ode, Comédie, Interrogatoire, Prière d'insérer, etc., soit des types façonnés par le comportement linguistique - souvent sociolinguistique - ou psychologique de l'énonciateur, tels Anglicismes, Paysan, Précieux, Vulgaire, Désinvolte, soit encore des textes rédigés dans un jargon «scientifique », comme Botanique, Zoologique, Géométrique ou Philosophique. Par ailleurs, dans certains de ces types de textes, on découvre encore des cas d'inventions lexicales qui n'ont pas d'influence décisive sur l'organisation informationnelle des phrases ; ainsi dans Médical, la scène devant la gare SaintLazare garde essentiellement la perspective fonctionnelle des phrases

\footnotetext{
${ }^{7}$ En parlant des «types de textes », je tiens compte des définitions à portée générale que l'on a données de cette notion. Je me réfère notamment à la définition d'Egon Werlich, qui considère les types de textes comme des «normes idéales » (idealtypische Normen) qui sont à la disposition du locuteur pour formuler linguistiquement les différents aspects de ses expériences (WERLICH, 1975 : 39). Cependant, il m'a semblé opportun d'adapter cette définition aux particularités de l'œuvre de Queneau, qui privilégie certains procédés assouplissant une typologie aux critères trop rigoureux.
} 
correspondantes dans Notations, pourtant formulées ici dans un jargon médical parodié : Plus tard, je le revois, hagard devant un Lazaret, en train de consulter un charlatan [...] (p. 57). De même, les textes rédigés dans un mélange de langues frappent plus par la créativité lexicale de l'auteur que par une réorganisation radicale des structures informationnelles; toujours cette scène devant la gare est présentée comme suit dans Hellénismes: En une chronie hystère, je l'esthèsis devant le sidérodromeux stathme hagiolazarique, péripatant [...] (p. 45).

En fin de compte, il se trouve - et c'est loin d'être surprenant - que les types de textes les plus intéressants pour les variations de la perspective fonctionnelle et de la progression thématique sont ceux qui mettent en jeu d'une part la grammaire (plus que le vocabulaire), d'autre part le langage reflétant l'attitude ou l'état psychologique de l'énonciateur. Ces aspects du texte peuvent bien être liés, mais pas nécessairement, à des genres codés ; ainsi par exemple, le texte intitulé Récit est tout particulièrement caractérisé par l'emploi du passé simple et la distinction des plans textuels; un autre texte, Télégraphique, supprime les déterminants et la plupart des prépositions, effaçant par là les frontières entre les constituants de la phrase ; dans un troisième texte, Lettre officielle, le style formel, associé à des situations de communication et à des rapports interpersonnels typiques, apparaît dans toute sa «splendeur ». Dans ce qui suit, je vais donc me concentrer, parmi les variantes du texte de départ, sur celles dont l'organisation informationnelle s'écarte nettement de la perspective fonctionnelle fondamentale, neutre, de Notations, et où ces écarts sont probablement déterminés par les types que représentent les textes particuliers. L'analyse suivra celle du texte modèle au début de cet article : je vais traiter d'abord les phénomènes phrastiques relatifs à la perspective fonctionnelle, aux positions et aux déplacements du thème et du rhème, pour terminer sur les problèmes de la progression thématique au niveau du texte.

\section{La perspective fonctionnelle}

Par rapport aux positions canoniques du thème et du rhème, les déplacements de ces constituants informationnels de la phrase donnent des tournures particulières aux textes qui les contiennent. Ces déplacements sont en général accompagnés de constructions grammaticales spécifiques et servent souvent à rendre emphatique l'expression du thème ou du rhème.

Ainsi, la thématisation d'un constituant de la phrase est normalement réalisée par des constructions segmentées qui peuvent disloquer le thème soit au début, soit à la fin d'une phrase ou d'une proposition. Courant dans le langage familier, ce procédé - avec dislocation à gauche - est employé dans Moi je, mais de manière exagérée, jusqu'à en perdre son rôle dans l'organisation des informations : [...] Deux heures plus tard, moi je rencontre de nouveau ce garçon. Moi, je l'aperçois devant la gare Saint-Lazare. Moi, je le vois en compagnie d'un copain de sa sorte qui lui disait, moi je l'ai entendu [...] Moi, je l'ai bien vu, il désignait le bouton supérieur (p. 25). En effet, cette insistance du locuteur sur sa propre personne, 
traduisant une habitude linguistique qui devient une sorte de tic, ${ }^{8}$ est susceptible d'agacer l'interlocuteur implicite ${ }^{9}$, qui aura de la peine à retenir les autres éléments du contenu. À son tour, la dislocation à droite apparaît de façon curieuse dans Animisme, où le chapeau du type, personnifié et devenant le héros de l'histoire, est évoqué régulièrement en fin de phrase: Puis il alla soudain s'asseoir, lui le chapeau (p. 14). Ce qui frappe dans cette construction, c'est le poids informationnel que possède le segment disloqué, car cette position, en principe réservée à un rappel du thème connu, est occupée ici par une expression dont le référent est d'abord perçu comme inattendu et surprenant - ces qualités étant plutôt liées au rhème. Évidemment, à mesure que le texte avance, le lecteur s'habitue à ce procédé, la surprise s'estompe ou même disparaît ; en revanche, cette «perte»sera compensée en quelque sorte par une attente grandissante de cette information, connue certes, mais dont le retour, à la manière d'un refrain, donne une certaine satisfaction esthétique au lecteur: Un ami lui conseillait de faire ajouter un bouton supplémentaire à son pardessus... un bouton supplémentaire... à son pardessus... lui dire ça... à lui... lui le chapeau (p. 15). ${ }^{10}$

Quant à la partie rhématique de la phrase ou de la proposition, les constructions semblent plus diversifiées, peut-être parce que le rhème peut contenir un nombre varié de constituants placés après le sujet thème. Or, là où le verbe conjugué ne serait qu'un élément de transition entre le thème et le rhème, il peut être supprimé, comme dans Télégraphique, la suppression étant en accord avec, ou même exigée par la brièveté conventionnelle de ce type de texte : Bus bondé stop [...] Question doigts pieds froissés contact talon prétendu volontaire stop (p. 41). ${ }^{11}$ Par contre-coup, les rares verbes conjugués maintenus attirent l'attention, et par là, ils tendent à devenir rhématiques : JNhomme abandonne discussion pour place libre stop (p. 41). La structure informationnelle d'Analyse logique est encore plus frappante, car tandis que dans les phrases du télégramme on pouvait encore reconnaître plus ou moins la division thème - rhème, ici la presque totalité de l' «analyse » consiste en dénominations, c'est-à-dire que des rhèmes relatifs à un domaine de contenu se succèdent sans thèmes, et lorsque le domaine - le 'lieu', le 'temps', l'’action' - se trouve épuisé, il est lui-même nommé sous forme de rhème d'une phrase introduite par la construction présentative c'est: Autobus. Plate-forme. Plate-forme d'autobus. C'est le lieu.

\footnotetext{
${ }^{8}$ Le cas de ce texte est particulier. Normalement, on observe, certes, une tendance à présenter une situation du point de vue du locuteur, comme perçue par celui-ci, mais cette tendance est moins spectaculaire que dans $M o i$ je, et elle reste sans emphase dans l'expression. Ainsi, face à une présentation à la troisième personne du type devant la gare Saint-Lazare, dans Notations: Il est avec un camarade qui lui dit [...] (p. 5), on a dans Passé indéfini : J'ai vu mon type qui discutait avec un copain (p. 21). Cf. à ce propos (pour l'anglais surtout) MATHESIUS (1964:61-64).

${ }^{9}$ C'est-à-dire, dans ce cas, le lecteur de l'œuvre.

${ }^{10}$ Il est certain que le chapeau, étant dans un rapport métonymique avec celui qui le porte, pourrait simplement représenter ici le jeune homme. Cependant, le titre Animisme suggère que cet objet s'affranchit de son porteur, ce que souligne également cette formulation : je le revis se déplaçant à quelque un mètre soixante-six au-dessus du sol (p. 14-15).

${ }^{11}$ Il est vrai que le texte de Notations contient également des constructions sans verbes conjugués, mais là, ces constructions nominales représentent des rhèmes secondaires, en apposition : Un type dans les vingt-six ans, chapeau mou [...], cou trop long [...] (p. 5).
} 
Midi. Environ. Environ midi. C'est le temps (p. 18). ${ }^{12}$ La même formule présentative, mais combinée cette fois avec la négation parallèle $n i .$. ni... suivie d'une coordination adversative mais... d'un bout à l'autre de Négativités, favorise tout particulièrement la mise en valeur du rhème par la force du contraste entre les rhèmes virtuels niés et le rhème affirmé : Ce n'était ni un bateau, ni un avion, mais un moyen de transport terrestre (p. 14). Tout comme la négation, l'interrogation porte nécessairement sur le rhème; ainsi, le début typique d'Interrogatoire, précisant les coordonnées des événements par la formule question - réponse, indique les circonstances dans des réponses elliptiques qui donnent juste l'essentiel de l'information attendue, c'est-à-dire le rhème propre dans la dynamique communicative : - À quelle heure ce jour-là passa l'autobus de la ligne $S$ de midi 23, direction porte de Champerret ? - À midi 38 (p. 28).

\section{La progression thématique}

Si dans la section précédente il était question uniquement des positions du thème et du rhème à l'intérieur de la phrase, c'est que les différentes structures syntaxiques et informationnelles identifiées se répètent avec insistance à travers la totalité, ou du moins une bonne partie, des textes, et cette répétition prime à tel point dans chacun des textes cités qu'il est impossible d'y découvrir des variations marquantes dans la progression thématique. En revanche, dans d'autres variantes du texte de départ, où les répétitions littérales se font plus rares, c'est la progression thématique qui prend un intérêt accru. Conditionnée par le type de texte - ici encore, codé ou non -, la progression thématique concourt à créer la tonalité et le style particuliers du texte.

Il semble que les variantes les plus proches de Notations soient celles qui se proposent de présenter la situation sous forme de récit (dans des textes de longueur à peu près égale), en suivant la chronologie naturelle, ${ }^{13}$ avec relativement peu d'«intrusions » subjectives du narrateur-témoin, ${ }^{14}$ ces deux facteurs permettant une progression thématique équilibrée, basée sur la progression à thème constant. Mais tandis que le récit prototypique, lui-même intitulé Récit, est d'une facture classique, faisant alterner le passé simple et l'imparfait : j'aperçus un personnage [...] qui portait un feutre mou (p. 13), les quatre textes rédigés chacun dans un seul temps verbal, Passé indéfini, Présent, Passé simple, Imparfait, montrent une progression plus mécanique; dans les deux derniers surtout, la distinction des

\footnotetext{
${ }^{12}$ Ailleurs, c'est la construction impersonnelle il y $a$, également à fonction présentative, qui introduit les longues énumérations de diverses odeurs - autant de rhèmes juxtaposés dans l'unique phrase complexe d'Olfactif: [...] il y avait une certaine senteur de long cou juvénile, une certaine perspiration de galon tressé, une certaine âcreté de rogne [...] (p. 38).

${ }^{13}$ Sauf Le côté subjectif, inversant l'ordre des scènes, et Rétrograde, qui commence par la mention du bouton, pour se terminer «sur la plate-forme de l'autobus ».

${ }^{14}$ Dans la plupart des textes du type récit - à commencer par Notations -, c'est un narrateur homodiégétique témoin qui raconte les événements. Cependant, dans Le côté subjectif, le narrateur homodiégétique est le protagoniste même (le type au chapeau). À son tour, la dernière variante, Inattendu, offre une troisième solution : dans cette scène dialoguée, introduite par un bref passage narratif présentant les personnages, c'est un narrateur intra-homodiégétique témoin qui raconte l'histoire. Or, à la fin du dialogue, le participant jusque-là silencieux se révèle comme ayant été l'un des acteurs des événements (l'ami).
} 
plans textuels - celui des événements principaux et celui des circonstances, des descriptions et des commentaires - se trouve effacée, et l'ensemble donne l'impression d'un exercice de grammaire (du type : «mettez à l'imparfait»), sans doute parodié : Dès qu'il apercevait une place libre, il se précipitait vers elle et s'y asseyait (p. 22). En revanche, représentant un autre type de texte, Prière d'insérer - soigneusement construite pour faire figurer le maximum d'informations dans un texte aux dimensions nécessairement réduites - distingue deux plans: l'un reproduit l'histoire sous forme de synopsis, l'autre, qui encadre cette dernière, commente l'œuvre et l'auteur dans un métalangage analytique, ayant souvent recours aux rhèmes secondaires, pour la concision : [le romancier] s'est appliqué à ne mettre en scène que des personnages bien dessinés et agissant dans une atmosphère compréhensible par tous ( $\mathrm{p}$. 17). Opposé au récit, le commentaire montre plutôt une progression à thèmes dérivés, tels personnages, intrigue, épisode final, et même romancier, reliés par anaphore associative à l'hyperthème roman, annoncé dès le début de cette argumentation.

Dans un assez grand nombre de variantes, le genre du récit subit des changements qui concernent d'une part la longueur des textes (et par conséquent l'abondance des détails), d'autre part la manifestation de diverses émotions du narrateur. Quant à la longueur, les textes oscillent entre les 31 syllabes du poème japonais, Tanka, et une page dense, pour Insistance. ${ }^{15}$ Ces latitudes ont naturellement un effet prévisible sur la progression thématique : plus le texte est court, plus le «tempo » est rapide pour une présentation aussi complète de l'histoire que possible. Ainsi, chaque vers de Tanka constitue une unité autonome, rhématique - ou si l'on veut, avec rupture thématique: L'autobus arrive / Un zazou à chapeau monte / Un heurt il y a / Plus tard devant Saint-Lazare / Il est question d'un bouton (p. 47). Même structure dans Vers libres, où la brièveté n'est pas dictée par le genre, mais choisie par l'auteur: L'autobus / plein / le cœur / vide / le cou / long / le ruban / tressé / les pieds / plats / plats et aplatis / la place / vide [...] (p. 47). ${ }^{16}$ Les problèmes de longueur apparaissent parfois liés à des figures de rhétorique. Ainsi, des textes courts, comme Litotes, par ses ellipses et le ton réservé : Nous étions quelques-uns à nous déplacer de conserve. Un jeune homme [...] (p. 6), ou Métaphoriquement, contenant à peine plus de mots, mais qui sont enrichis par les expressions imagées: Au centre du jour, jeté dans le tas des sardines voyageuses d'un coléoptère à l'abdomen blanchâtre, un poulet [...] (p.6), s'opposent à des textes amplifiés de diverses manières, tels Ampoulé, inspiré par le style de l'épopée antique : À l'heure où commencent à se gercer les doigts roses de l'aurore [...] (p. 27), Précieux, recherché et raffiné, où le bus est décrit comme ce chef-d'œuvre de l'industrie automobile française (p. 63), Définitionnel, d'un style formel rigoureux, selon lequel le bus est un grand véhicule public de transport urbain (p. 46), ou Précisions, remplies d'indications

\footnotetext{
${ }^{15}$ Certains textes sont plus longs en apparence, tels Ode, Comédie et Inattendu, mais comme ces textes représentent d'autres genres (ayant des conventions typographiques différentes), leurs pages plus aérées contiennent moins de mots.

${ }^{16} \mathrm{Il}$ y a là une organisation thème-rhème régulière et remarquable : un SN défini thématique ou du moins thématisé, un prédicat rhématique au «vers » suivant, avec en effet à chaque "paire » une rupture thématique ou sous-thématique.
} 
méticuleuses de mesures chiffrées: 118 minutes plus tard, il se trouvait à 10 mètres de la gare Saint-Lazare, entrée banlieue [...] (p. 11).

Tandis que ces textes amplifiés, donc plutôt longs, gardent tous un «tempo » relativement lent, mais sans pauses considérables dans la présentation des événements, un dernier groupe de textes montre des déviations marquées, traduisant des mouvements psychologiques qui empêchent le récit d'avancer. En effet, Rêve baigne dans le flou: Il me semblait que tout fût brumeux et nacré autour de moi, avec des présences multiples et indistinctes [...] (p. 8); dans Apartés, la narration se heurte à des remarques subjectives : L'autobus arriva [...] veine il y a encore une place pour moi (p. 31); Hésitations ne tranchent pas entre les rhèmes virtuels proposés : Je ne sais pas très bien où ça se passait... dans une église, une poubelle, un charnier? Un autobus peut-être? (p. 10); enfin, certains textes, avec leurs répétitions et leurs divagations interminables, de même qu'avec leur circularité, illustrent à merveille les difficultés de la narration, tels Insistance : Un jour, vers midi, je montai dans un autobus presque complet de la ligne S. Dans un autobus presque complet de la ligne $S$, il y avait un jeune homme assez ridicule. Je montai dans le même autobus que lui, et ce jeune homme, monté avant moi dans ce même autobus de la ligne $S$, presque complet, vers midi, portait sur la tête un chapeau que je trouvai bien ridicule [...], et le jeune homme qui le portait, ce chapeau, [...] se trouvait dans le même autobus que moi, un autobus presque complet parce qu'il était midi [...] (p. 19) ou, pour terminer, Maladroit, texte qui ne manque ni d'ironie, ni d'esprit ludique : Je n'ai pas l'habitude d'écrire. Je ne sais pas. [...] car je n'ai pas l'habitude d'écrire. Merde, je ne sais pas comment j'ai fait mais me voilà revenu tout au début. Je ne vais jamais en sortir. Tant pis. Prenons le taureau par les cornes. Encore une platitude. Et puis ce gars-là n'avait rien d'un taureau. Tiens, elle n'est pas mauvaise celle-là. Si j'écrivais [...] Peutêtre bien que ça me ferait connaître des messieurs de l'Académie française, du Flore et de la rue Sébastien-Bottin. Pourquoi ne ferais-je pas de progrès après tout. C'est en écrivant qu'on devient écriveron (p. 34-35).

\section{Conclusion}

Ce choix de textes a dû montrer, sur le plan linguistique, l'interdépendance entre la perspective fonctionnelle de la phrase et les types de textes. Il se trouve qu'à la perspective fonctionnelle de la phrase on puisse superposer une autre problématique, celle des types-genres textuels ; en effet, ces derniers déterminent au moins en partie - l'organisation informationnelle des phrases et avec cela, la progression thématique dans le texte. Parmi les facteurs organisationnels, les plus importants sont sans doute la longueur relative des textes et le rapport entre longueur et «tempo», le caractère codé ou non du genre représenté, le comportement (socio)linguistique ou psychologique du locuteur influençant la production du texte, et le caractère rhétorique et/ou ludique de la formulation. Enfin, sur le plan littéraire, tous ces jeux des Exercices de style préfigurent l'activité du futur Oulipo, dont les procédés d'écriture sont traités par Queneau « avec le brio qui lui est propre » (Prière d'insérer, p. 17). 


\section{BIBLIOGRAPHIE}

Texte analysé

QUENEAU Raymond (2006), Exercices de style [1947], in : Euvres complètes III : Romans II (publ. sous la dir. d'Henri Godard), Paris, Gallimard, Bibl. de la Pléiade, p. 3-65, et p. 1547-1582 (pour les « Notices, notes et variantes », établies par Emmanuël Souchier).

\section{Références}

COMBETTES Bernard (1986), Introduction et reprise des éléments d'un texte, Pratiques 49, p. 69-84.

DANEŠ František (1974), Functional Sentence Perspective and the Organization of the Text, in: Daneš F. (ed.), Papers on Functional Sentence Perspective, Prague, Academia - The Hague-Paris, Mouton, Janua Linguarum, Series Minor 147, p. 106-128.

FIRBAS Jan (1971), On the Concept of Communicative Dynamism in the Theory of Functional Sentence Perspective, Sborník Prací Filosofické Fakulty Brněnské University, Series Linguistica A 19, p. 135-144.

FIRBAS Jan (1974), Some Aspects of the Czechoslovak Approach to Problems of Functional Sentence Perspective, in : Daneš F. (ed.), Papers on Functional Sentence Perspective, Prague, Academia - The Hague-Paris, Mouton, Janua Linguarum, Series Minor 147, p. 11-37.

MATHESIUS Vilém (1964), On Linguistic Characterology with Illustrations from Modern English [1928], in : Vachek Josef (ed.), A Prague School Reader in Linguistics, Bloomington, Indiana University Press, p. 59-67.

WERLICH Egon (1975), Typologie der Texte, Heidelberg, Quelle \& Meyer. 